\title{
Flow resistivity estimation from practical absorption coefficients of fibrous absorbers
}

\author{
Jeong, Cheol-Ho
}

Published in:

Applied Acoustics

Link to article, DOI:

10.1016/j.apacoust.2019.107014

Publication date:

2020

Document Version

Peer reviewed version

Link back to DTU Orbit

Citation (APA):

Jeong, C-H. (2020). Flow resistivity estimation from practical absorption coefficients of fibrous absorbers. Applied Acoustics, 158, [107014]. https://doi.org/10.1016/j.apacoust.2019.107014

\section{General rights}

Copyright and moral rights for the publications made accessible in the public portal are retained by the authors and/or other copyright owners and it is a condition of accessing publications that users recognise and abide by the legal requirements associated with these rights.

- Users may download and print one copy of any publication from the public portal for the purpose of private study or research.

- You may not further distribute the material or use it for any profit-making activity or commercial gain

- You may freely distribute the URL identifying the publication in the public portal

If you believe that this document breaches copyright please contact us providing details, and we will remove access to the work immediately and investigate your claim 
Flow resistivity estimation from practical absorption coefficients of fibrous absorbers.

\section{Cheol-Ho Jeong}

6

Acoustic Technology, Technical University of Denmark, 2800 Kongens Lyngby, Denmark 


\section{Abstract}

9 There are useful conversion methods from Sabine absorption coefficients according to ISO 354 to 10 other acoustic properties for room boundaries, e.g., surface impedance or flow resistivity. However, 11 most available sound absorption coefficients are practical absorption coefficients, which are simplified according to ISO 11654 from the Sabine absorption coefficients and widely used by absorber manufacturers as their absorbers' performance indicators. In this study, practical absorption coefficients are used to inversely characterize the flow resistivity via reliable models. 15 absorber samples with varying mounting conditions are used for validating the flow resistivity estimation, having a wide flow resistivity range of 10 to $110 \mathrm{kNsm}^{-4}$. As expected, the practical absorption coefficients are found to be less reliable input parameters for estimating the flow resistivity, but with more datasets, the degradation becomes insignificant compared to the Sabine absorption coefficients. The estimated flow resistivity will be a valuable parameter to predict the absorption characteristics of different thickness and mounting conditions.

Keywords: flow resistivity; fibrous materials; Sabine absorption coefficients; practical absorption coefficient; inverse characterization 


\section{Introduction}

The absorption properties of sound absorbers are mostly characterized in reverberation chambers by measuring two sets of the reverberation times of an empty and occupied condition, resulting in the Sabine absorption coefficients, $\alpha_{\mathrm{Sab}}$, in the 18 one-third octave bands centered from $100 \mathrm{~Hz}$ to $5000 \mathrm{~Hz}$ [1]. The 18 absorption coefficients in the one-third octave bands are averaged into the corresponding octave bands and further simplified by truncations and rounding, resulting in the practical absorption coefficient, $\alpha_{\mathrm{p}}$ [2]. The main reason for the truncation is the fact that the Sabine absorption coefficients can be higher than unity due to the finiteness of a sample $[3,4]$ and individual chambers' non-diffuse condition [5-11], which is unphysical.

Manufactures of sound absorbers normally prefer simplified metrics, such as practical absorption coefficients or single-value ratings based on the practical absorption coefficients that are called the weighted sound absorption coefficients [2]. Practical absorption coefficients can be used in room acoustic simulations to represent the acoustic properties of the boundary surfaces, but only possible in energy-based geometrical acoustics simulations. In wave-based methods and phased geometrical methods, complex-valued coefficients, e.g., surface impedance or pressure reflection coefficients, are needed. Therefore, some useful conversion methods have been suggested in the literature [12-14]. In this study, the estimation of the flow resistivity, $\sigma$, is of the main interest, particularly using the practical absorption coefficients as input data. This study uses four different absorption models to compare their performances in extracting the flow resistivity, together with two input absorption data sets, $\alpha_{\mathrm{Sab}}$ and $\alpha_{\mathrm{p}}$. Once the flow resistivity is extracted, this information can be useful to estimate the absorption properties of different thickness, different air backing conditions, and other multi-layered absorber configurations, which the absorber manufacturers do not provide directly.

\section{Methods}

Four flow resistivity estimation models are tested using 15 absorption coefficient datasets. The four models consist of two finite size corrections and two different ways to account for the room's influence on the absorption coefficients. The measured flow resistivity information of the tested absorbers 
according to Method A in ISO 29053 [15] is provided by the manufacturer, SG Ecophon. Note that all the absorption coefficients are measured in the same reverberation chamber.

\section{1. Absorption models}

Four absorption models that can account for the finiteness of the samples and reverberation chambers' effects are used and more details of the models can be found in Refs. $[9,10,14]$. The first model, named as Model 1, uses the radiation impedance suggested by Thomasson [3] with a frequency-dependent room correction [10] and Model 2 has a frequency-independent room compensation [9] as shown in Eq. 1.

$$
\begin{aligned}
& \alpha_{\text {Thomasson }}(f)=2 \int_{0}^{\pi / 2} \frac{4 \operatorname{Re}\left(Z_{w}(f, \theta)\right)}{\left|Z_{w}(f, \theta)+\bar{Z}_{r}(f, \theta)\right|^{2}} \sin (\theta) d \theta, \\
& \alpha_{M 1}(f)=\alpha_{\text {Thomasson }}(f)+\alpha_{\text {room } 1} \frac{R S D(f) \cdot \alpha_{\text {Thomasson }}(f)}{\left[R S D(f) \cdot \alpha_{\text {Thomasson }}(f)\right]}, \\
& \alpha_{M 2}(f)=\alpha_{\text {Thomasson }}(f)+\alpha_{\text {room } 2} .
\end{aligned}
$$

Here, $Z_{w}$ is the surface impedance as a function of frequency and angle of incidence, $\theta$, and $\bar{Z}_{r}(f, \theta)$ is the average radiation impedance of a finite specimen over the azimuth angle derived by Thomasson, see more details in Ref. [3]. The frequency-independent room factor in Model 2 is the simplest way to compensate for the influence of the room on the Sabine absorption coefficient, first suggested in Ref [9]. Later, it was modified to include the relative standard deviations (RSD) of two different absorption data measured from 13 chambers in an absorption round robin test [16], which means that the reverberation chamber under test follows the average behavior of spectral fluctuation observed in the 13 chambers in the round robin test. RSD is used as the normalized, predefined, frequency-dependent effect of the test chamber on the measured absorption, with $[\bullet]$ being the average across the frequency of interest. If the reverberation chamber under test behaves similarly to the average characteristics of the 13 chambers, it is likely that the frequency-dependent room correction, i.e., Model 1, should outperform the frequency-independent correction, Model 2, as shown in Ref [10]. Note $\alpha_{\text {room1 }}$ and $\alpha_{\text {room2 }}$ are the optimization parameters for Model 1 and Model 2, respectively. 
This original Thomasson's finite size radiation impedance was derived to account for the finiteness of an absorber sample under two important assumptions: infinite baffle and flush mounting of an absorber sample [3]. These two assumptions are hard to achieve in usual ISO 354 measurement settings, as the sample size is restricted between 10 and $12 \mathrm{~m}^{2}$ and a flush mounting can never be fulfilled in any laboratories. The thinner the sample, the closer to the flush mounting assumption. Recently, it was found that Thomasson's correction for sound absorption works best for smaller surfaces and thinner materials with a brief guideline of sample sizes smaller than $4 \mathrm{~m}^{2}$ and sample thicknesses thinner than $5 \mathrm{~cm}$, but fails to predict measured data when the sample gets larger and thicker [14]. This is particularly problematic when the absorber is measured with an air cavity backing, making the overall depth of the test absorber thicker. For large thicknesses typically including an air cavity, a model using the complex conjugate of Thomasson's radiation impedance of is found to outperform [14], which is a basis for Model 3 and 4. Model 3 uses the frequency dependent room correction, while a frequency independent room factor is used in Model 4. The room correction is closely related to the degree of non-diffusion in the reverberation chambers, which is an important current research topic [11,17-19].

$$
\begin{aligned}
& \alpha_{\text {size }}(f)=2 \int_{0}^{\pi / 2} \frac{4 \operatorname{Re}\left(Z_{w}(f, \theta)\right)}{\left|Z_{w}(f, \theta)+\bar{Z}_{r}^{*}(f, \theta)\right|^{2}} \sin (\theta) d \theta, \\
& \alpha_{M 3}(f)=\alpha_{\text {size }}(f)+\alpha_{\text {room } 3} \frac{R S D(f) \cdot \alpha_{\text {size }}(f)}{\left[R S D(f) \cdot \alpha_{\text {size }}(f)\right]} \\
& \alpha_{M 4}(f)=\alpha_{\text {size }}(f)+\alpha_{\text {room } 4} .
\end{aligned}
$$

Here $\alpha_{\text {size }}$ is the size-corrected absorption coefficient using the complex conjugate of Thomasson's radiation impedance, $\alpha_{\text {room3 }}$ and $\alpha_{\text {room4 }}$ are the optimization parameters for Model 3 and Model 4, respectively.

The most likely flow resistivity, $\sigma_{\text {est }}$, is found by minimizing the cost function as follows

$$
e\left(\sigma, \alpha_{\text {room }}\right)=\sum_{f=f_{\min }}^{f_{\max }}\left|\alpha_{\text {input }}(f)-\alpha_{M}\left(\sigma, \alpha_{\text {room }}, f\right)\right|,
$$

where $\alpha_{\text {input }}$ represents the input absorption data for the flow resistivity extraction, either $\alpha_{\text {Sab }}$ or $\alpha_{\mathrm{p}}$.

\subsection{Absorption coefficient datasets, $\alpha_{\text {Sab }}$ or $\alpha_{p}$}


Ecophon SG provided 15 sets of the Sabine absorption coefficient data obtained by ISO 354 measurements of five glasswool materials, each having three different backing conditions. Note the 101 three backing conditions are not identical for all the materials. A summary of the backing conditions and flow resistivity is shown in Table 1 . The Sabine absorption coefficients of 15 cases are shown in Fig 1. Short names of the scenarios are made in the form of $A_{n} B_{m}$, the first subscript, $n$, being the glasswool material number, and the second subscript, m, representing the backing condition.

105
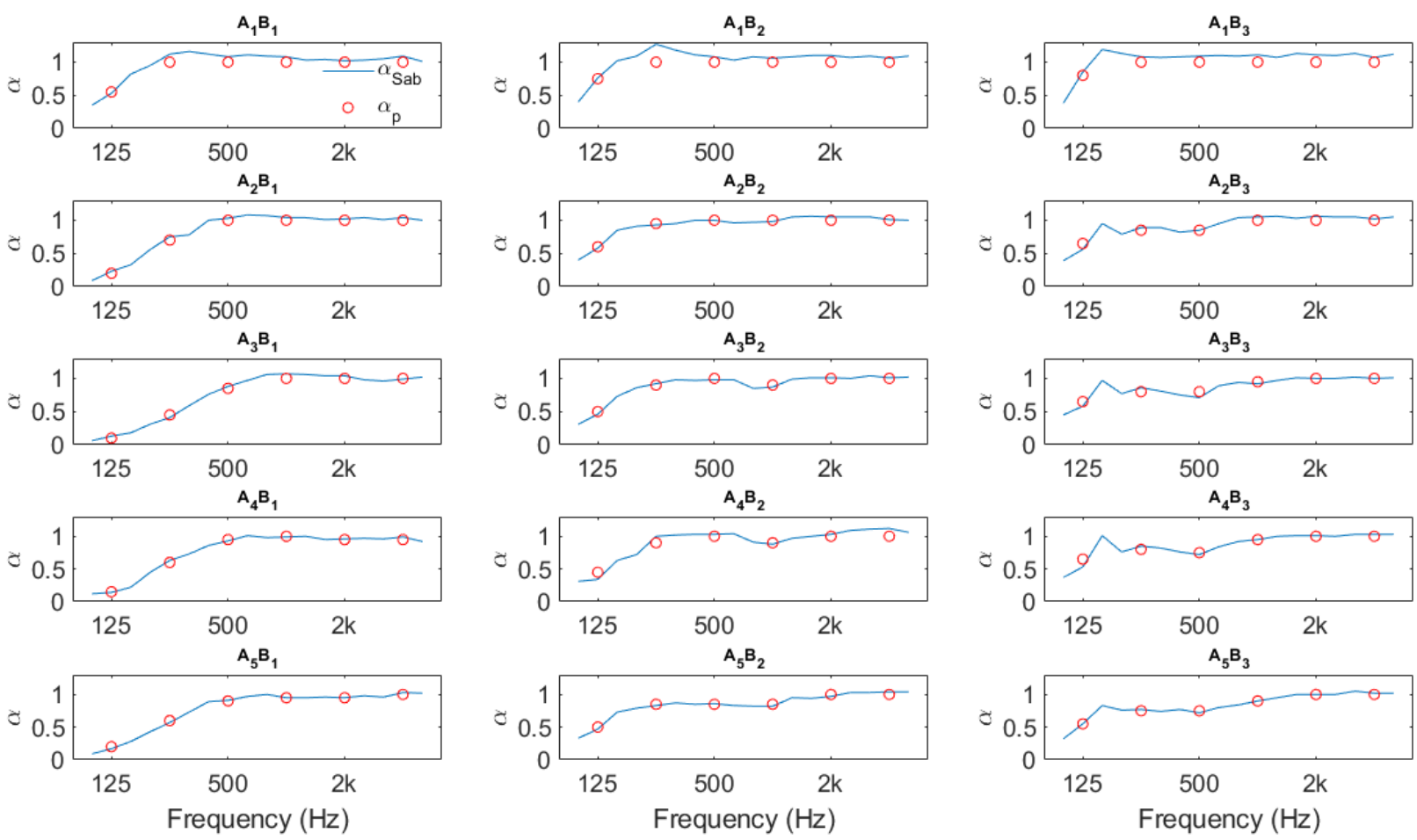

106

107 Figure. 1. Sabine and practical absorption coefficients of the absorbers tested. (Color in electronic version only) 

tested.

\begin{tabular}{|c|c|c|c|}
\hline Absorber/Backing & Absorber thickness (mm) & Overall depth of system (mm) & $\sigma_{\text {meas }}\left(\mathrm{kNs} / \mathrm{m}^{4}\right)$ \\
\hline $\mathrm{A}_{1} \mathrm{~B}_{1}$ & 100 & 100 & 10.90 \\
\hline $\mathrm{A}_{1} \mathrm{~B}_{2}$ & 100 & 200 & 10.90 \\
\hline $\mathrm{A}_{1} \mathrm{~B}_{3}$ & 100 & 400 & 10.90 \\
\hline $\mathrm{A}_{2} \mathrm{~B}_{1}$ & 40 & 50 & 39.26 \\
\hline $\mathrm{A}_{2} \mathrm{~B}_{2}$ & 40 & 200 & 39.26 \\
\hline $\mathrm{A}_{2} \mathrm{~B}_{3}$ & 40 & 400 & 39.26 \\
\hline $\mathrm{A}_{3} \mathrm{~B}_{1}$ & 20 & 50 & 62.93 \\
\hline $\mathrm{A}_{3} \mathrm{~B}_{2}$ & 20 & 200 & 62.93 \\
\hline $\mathrm{A}_{3} \mathrm{~B}_{3}$ & 20 & 400 & 62.93 \\
\hline $\mathrm{A}_{4} \mathrm{~B}_{1}$ & 20 & 60 & 80.64 \\
\hline $\mathrm{A}_{4} \mathrm{~B}_{2}$ & 20 & 200 & 80.64 \\
\hline $\mathrm{A}_{4} \mathrm{~B}_{3}$ & 20 & 400 & 80.64 \\
\hline $\mathrm{A}_{5} \mathrm{~B}_{1}$ & 20 & 65 & 105.69 \\
\hline $\mathrm{A}_{5} \mathrm{~B}_{2}$ & 20 & 200 & 105.69 \\
\hline $\mathrm{A}_{5} \mathrm{~B}_{3}$ & 20 & 400 & 105.69 \\
\hline
\end{tabular}

112

116 (1) The 18 Sabine absorption data in the $1 / 3$ octave bands are arithmetically averaged into the 6 octave

Most absorber manufacturers provide practical absorption coefficient datasets. The procedures to calculate the practical absorption coefficient is as follows [2].

117 bands centered from $125 \mathrm{~Hz}$ to $4 \mathrm{kHz}$.

118 (2) The $1 / 1$ octave band absorption coefficients are rounded in steps of 0.05.

119 (3) If the rounded absorption coefficient is higher than 1 , then the value is maximized to 1. 

coefficient, particularly in the shape of the absorption curve. This spectral shape of the absorption data is known to help identify the flow resistivity when the backing cavity is used [10]. Particularly for the $\mathrm{B}_{3}$ conditions, there is a consistent peak at $160 \mathrm{~Hz}$ in the Sabine absorption coefficients, but disappeared in the practical absorption coefficient due to the truncation and averaging process.

\subsection{Optimization procedure for $\sigma$ and $\alpha_{\text {room }}$.}

The whole calculation procedure is summarized in Fig. 2, where the four absorption models with two input data, the Sabine and practical absorption coefficient, are compared. As this is a two dimensional optimization problem to find the most likely flow resistivity and room's effect on the measured absorption coefficient, the error in Eq. (3) was evaluated for a typical range of the flow resistivity from 1000 to $200000 \mathrm{Nsm}^{-4}$ with steps of $500 \mathrm{Nsm}^{-4}$, and $\alpha_{\text {room }}$ is searched from -0.3 to 0.3 with steps of 0.005 . Note that there have been other optimization techniques investigated using, e.g., simplex optimization [9] or Bayesian optimization [20].

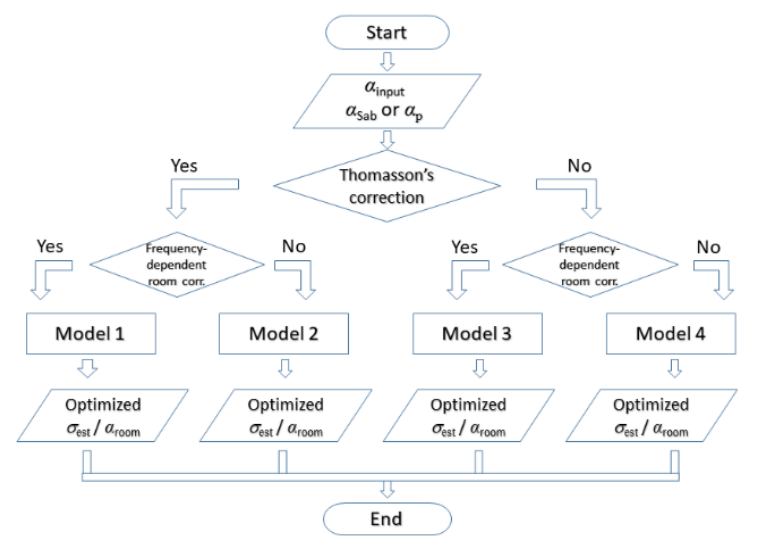

Figure 2. Flow chart for the flow resistivity estimation. (Color in electronic version only)

\section{Results}

Figure 3 compares the extracted flow resistivity and measured flow resistivity using the Sabine absorption coefficient. The size of the symbol is proportional to the error function value in Eq. (3), meaning that the smaller the symbol, the smaller the difference between the input absorption coefficient and resulting absorption coefficient. 

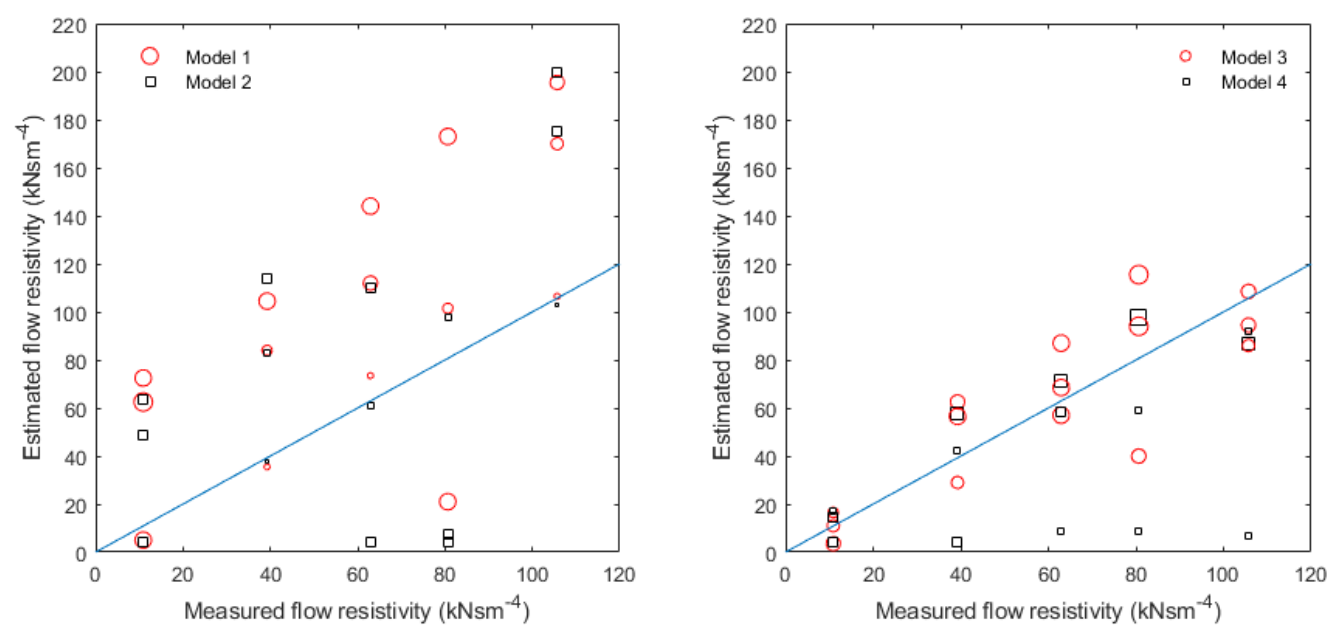

Figure 3. Comparison of measured and estimated flow resistivity using the Sabine absorption coefficients. (Color in electronic version only)

Generally speaking, using the Thomasson's original correction in Model 1 and 2, the extracted flow resistivity deviates quite a lot from the measured flow resistivity, but there are a few cases where Model 1 and 2 significantly outperform the others, Model 3 and 4 . These are the cases with relatively small overall depths, such as $5 \mathrm{~cm}$ with $\mathrm{A}_{2} \mathrm{~B}_{1}$ and $\mathrm{A}_{3} \mathrm{~B}_{1}, 6 \mathrm{~cm}$ with $\mathrm{A}_{4} \mathrm{~B}_{1}$. This confirms that Thomasson's model successfully replicate the measured Sabine absorption for an overall thickness smaller than about $6 \mathrm{~cm}$, which concurs with the previous finding [14]. For overall depths larger than $6 \mathrm{~cm}$, the results get inaccurate with Model 1 and 2, mostly overestimating the flow resistivity values. With $\delta$ defined as $\left(\sigma_{\text {est }}\right.$ - $\left.\sigma_{\text {meas }}\right)$, the averaged $\delta$ values across the 15 absorber cases using Model 1 and 2 are $37 \mathrm{kNsm}^{-4}$ and 14 $\mathrm{kNsm}^{-4}$, with standard deviation of $43 \mathrm{kNsm}^{-4}$ and $53 \mathrm{kNsm}^{-4}$, respectively. Here, both the average and standard deviation of $\delta$ are used as performance indicators, as the average value of $\delta$ indicates the closeness to the true flow resistivity on an overall sense, while the standard deviation refers to the precision of the estimation, quantifying the closeness of agreement among a set of the estimated flow resistivity. The standard deviation is also related to the reliability of the extraction method, as a big spread of the estimated flow resistivity is not ideal. Note that Model 1 and 2 yield unacceptably large averages and standard deviations.

In Fig. 3, Model 3 and 4 predict the flow resistivity values more accurately than Model 1 and 2. Using Model 4 with the frequency-independent room correction, there is always one mounting condition for each material that produces an acceptably large $\delta$, which is found to be always the largest 
overall depth cases of $40 \mathrm{~cm}$ in the cases of $\mathrm{A}_{2} \mathrm{~B}_{3}, \mathrm{~A}_{3} \mathrm{~B}_{3}, \mathrm{~A}_{4} \mathrm{~B}_{3}$ and $\mathrm{A}_{5} \mathrm{~B}_{3}$. With the frequency dependent room correction, Model 3, the flow resistivity estimation gets most accurate on average. The average difference $\delta$ of the flow resistivity using Model 3 is $2 \mathrm{kNsm}^{-4}$, whereas Model 4 yields $-17 \mathrm{kNsm}^{-4}$. The standard deviations are $19 \mathrm{kNsm}^{-4}$ and $34 \mathrm{kNsm}^{-4}$ for Model 3 and 4, respectively. Using Model 3, the mean and standard deviation of $\delta$ are smallest of all.

Figure 4 compares the extracted flow resistivity and measured flow resistivity using the practical absorption coefficient dataset. Although the shapes of the practical absorption curves get quite changed from those of the Sabine absorption due to substantial simplifications, the estimated flow resistivity is surprisingly not too much degraded with certain models. Generally speaking, $\alpha_{p}$ does not perform as good as $\alpha_{S a b}$, but unexpected improvements are also observed in some cases. Using Model 1 and 2, the estimated flow resistivity values are still quite overestimated with the average $\delta$ of $34 \mathrm{kNsm}^{-4}$ and 50 $\mathrm{kNsm}^{-4}$, respectively. The standard deviations, however, are reduced to $19 \mathrm{kNsm}^{-4}$ and $34 \mathrm{kNsm}^{-4}$ compared to the values obtained using Sabine absorption coefficient, $43 \mathrm{kNsm}^{-4}$ and $53 \mathrm{kNsm}^{-4}$, respectively. Similarly for Model 4, the average standard deviation in the estimated flow resistivity becomes $22 \mathrm{kNsm}^{-4}$, whereas the standard deviation is $34 \mathrm{kNsm}^{-4}$ using $\alpha_{S a b}$. For Model 3, $\alpha_{p}$ produces consistently more inaccurate and imprecise results than $\alpha_{S a b}$. All the means and standard deviations of $\delta$ for Model 3 and 4 can be found in Table 2.
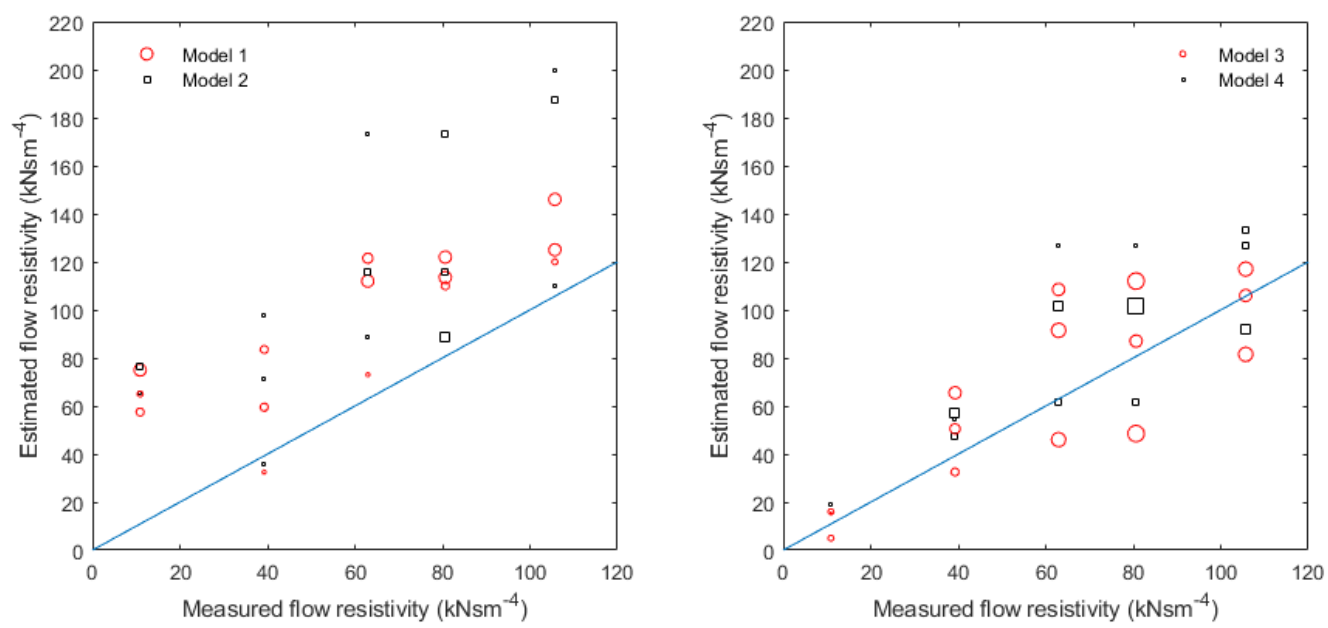

Figure 4. Comparison of measured and estimated flow resistivity using the practical absorption coefficients. (Color in electronic version only) 
Figure 5 summarizes the statistics of $\delta$ in using Sabine and practical absorption coefficients. Clearly,

185 Model 3 outperforms the others in all cases with the smallest average and standard deviation of $\delta$. The extracted flow resistivity using Model 4 varies quite a lot depending on the input data $\left(-17 \mathrm{kNsm}^{-4}\right.$ from the Sabine absorption to $17 \mathrm{kNsm}^{-4}$ from the practical absorption). The standard deviation is generally reduced with $\alpha_{\mathrm{p}}$ except for Model 3.

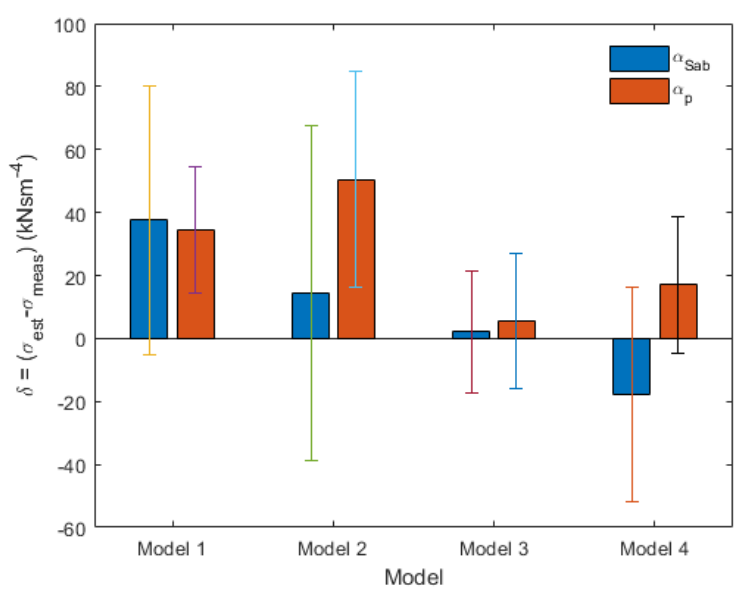

Figure 5. The statistics of the difference between the estimated and measured flow resistivity. (Color in electronic version only)

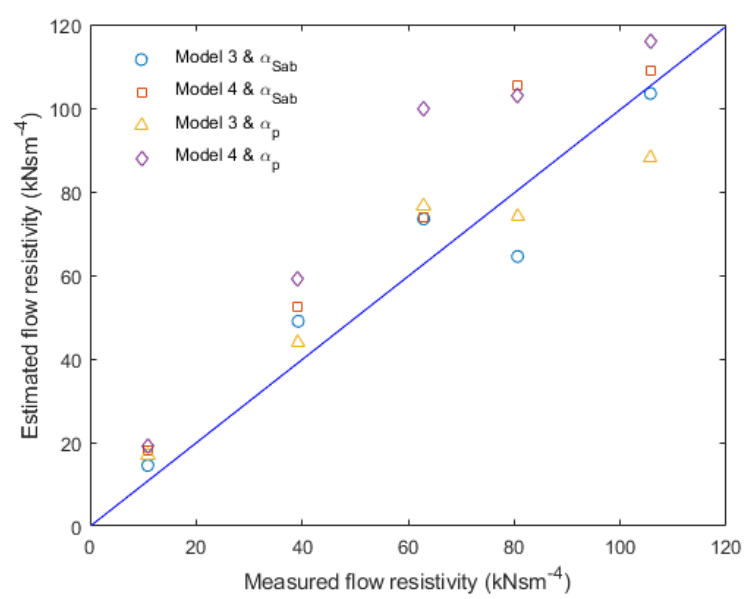

193 Figure 6. Comparison of measured and estimated flow resistivity for a single optimization using all the backing conditions for each absorber. (Color in electronic version only) 
In principle, no matter what the backing condition is, the flow resistivity should be the same for each absorber. Therefore, another optimization is tested by changing the cost function in Eq. (3) such that one flow resistivity per absorber type is estimated using all the three mounting datasets.

$$
e\left(\sigma, \alpha_{\text {room }}\right)=\sum_{m c=1}^{k} \sum_{f=f_{\min }}^{f_{\max }}\left|\alpha_{i n p u t, m c}(f)-\alpha_{M, m c}\left(\sigma, \alpha_{\text {room }}, f\right)\right|
$$

Here, $m c$ represents the mounting condition and $k$ means the number of available mounting conditions, which is always three in the present study. In practice, absorber manufacturers indeed provide the absorption characteristics for at least a couple of mounting conditions per absorber, so one could estimate the flow resistivity by adding all available backing conditions into a single optimization routine using the cost function in Eq. (4). The optimization results based on Eq. (4) are shown in Fig. 6 only for Model 3 and 4, as Model 1 and 2 systematically yield overestimations larger than $40 \mathrm{kNs} / \mathrm{m}^{4}$. The estimated flow resistivity values based on Eq. (4) are more accurate than those based on Eq. (3) shown in Figs. 3-5. A comparison of the mean and standard deviation of $\delta$ between the individual absorber/mounting estimation and grouped optimization is summarized in Table 2, resulting in a significant reduction in the standard deviation by the grouped optimization - almost halved in all conditions -, which means the precision of the estimation is significantly improved when more datasets are combined into a single optimization. In terms of the mean difference, certain improvements are seen except for Model 4 using $\alpha_{\mathrm{p}}$. Therefore, it is concluded that the grouped optimization is likely to improve the flow resistivity estimation as the use of all available data prevents from producing overly large deviations mainly for large cavity mountings.

\section{Conclusion}

This study is concerned with the flow resistivity estimation using the Sabine absorption coefficient and practical absorption coefficient. The best model for flow resistivity extraction in terms of precision and accuracy is found to be Model 3, with the complex conjugated radiation impedance and frequency dependent room correction. However, the Thomasson's original model works best for thinner samples, when the original assumption is satisfied. The frequency independent room correction can give overly erroneous results in large air backing conditions. Surprisingly the use of practical absorption coefficient 
does not degrade the quality of the estimated flow resistivity signify cantly. Finally, an optimization

225 using all available mounting conditions in one single routine is found to estimate the flow resistivity

226 better in terms of accuracy and precision.

227

\section{Acknowledgements}

229

The author is grateful to Erling Nilsson, Ecophon SG, who provided the absorption data for this research.

Table 2. Summary of the statistics for the flow resistivity difference $\delta$ in $\mathrm{kNsm}^{-4}$. Std means the standard deviation.

\begin{tabular}{|c|c|c|c|c|c|c|c|c|}
\hline \multirow{4}{*}{ Model3 } & \multicolumn{4}{|c|}{ Individual optimization } & \multicolumn{4}{|c|}{ Grouped optimization } \\
\hline & \multicolumn{2}{|c|}{$\alpha_{\mathrm{Sab}}$} & \multicolumn{2}{|c|}{$\alpha_{\mathrm{p}}$} & \multicolumn{2}{|c|}{$\alpha_{\mathrm{Sab}}$} & \multicolumn{2}{|c|}{$\alpha_{\mathrm{p}}$} \\
\hline & mean & Std & mean & Std & mean & Std & mean & Std \\
\hline & 2.1 & 19.2 & 5.6 & 21.4 & 1.1 & 10.9 & 0.1 & 12.2 \\
\hline \multirow{4}{*}{ Model4 } & \multicolumn{4}{|c|}{ Individual optimization } & \multicolumn{4}{|c|}{ Grouped optimization } \\
\hline & \multicolumn{2}{|c|}{$\alpha_{\mathrm{Sab}}$} & \multicolumn{2}{|c|}{$\alpha_{\mathrm{p}}$} & \multicolumn{2}{|c|}{$\alpha_{\mathrm{Sab}}$} & \multicolumn{2}{|c|}{$\alpha_{\mathrm{p}}$} \\
\hline & mean & Std & mean & Std & mean & Std & mean & Std \\
\hline & -17.8 & 34.2 & 17.0 & 21.6 & 11.9 & 8.1 & 19.5 & 11.52 \\
\hline
\end{tabular}


237 [1] ISO standard 354. Measurement of sound absorption in a reverberation room, International

238 Organization for Standardization, Geneva, Switzerland.

239 [2] ISO standard 11654 Sound absorbers for use in buildings - Rating of sound absorption, international Organization for Standardization, Geneva, Switzerland.

[3] S.-I. Thomasson, Theory and experiment on the sound absorption as function of the area, Tech. Rep. No. TRITA-TAK 8201, KTH, Stockholm, Sweden, 1982.

[4] Holmberg D, Hammer P, Nilsson E. Absorption and radiation impedance of finite absorbing patches. Acta Acust. Acust. 2003;89:406-415.

[5] Jeong C-H. A correction of random incidence absorption coefficients for the angular distribution of acoustic energy under measurement conditions. J. Acoust. Soc. Am. 2009;125:2064-2071. [6] Jeong C-H. Non-uniform sound intensity distributions when measuring absorption coefficients in reverberation chambers using a phased beam tracing. J. Acoust. Soc. Am. 2010;127: 3560-3568. [7] Kang H.-J, Ih J.-G.,Kim J.-S., Kim H.-S. An experimental investigation on the directional distribution of incident energy for the prediction of sound transmission loss. Appl. Acoust. 2002;63: 283-294.

[8] Makita Y, Fujiwara K. Effects of precision of a reverberant absorption coefficient of a plane absorber due to anisotropy of sound energy flow in a reverberation room. Acustica 39:1978:331-335. [9] Jeong C-H., Chang J.-H. Reproducibility of the random incidence absorption coefficient converted from the Sabine absorption coefficient. Acta Acust. Acust. 2015;101:99-112. [10] Jeong C.-H. Predicting the Sabine absorption coefficients of fibrous absorbers under various air backing conditions with a frequency-dependent diffuseness correction. J. Acoust. Soc. Am. 2016;140: $1498-1501$.

[11] Nolan M, Fernandez-Grande E, Brunskog J, Jeong C.-H. A wavenumber approach to quantifying the isotropy of the sound field in reverberant spaces," J. Acoust. Soc. Am. 2018;143: 2514-2526. [12] J. H. Rindel, An impedance model for estimating the complex pressure reflection factor, in Proc. Forum Acusticum 2011, Aalborg, Denmark, 2011. 
263 [13] Mondet B, Brunskog J, Jeong C.-H., Rindel JH. From absorption to impedance: enhancing

264 boundary conditions in room acoustic simulations. Appl. Acoust. In press, 2019.

265 [14] Jeong C.-H. Sabine absorption coefficient predictions using different radiation impedances of a 266 finite absorber. Acta Acust. Acust. 2015;101:663-667.

267 [15] ISO standard 29053. Materials for acoustical applications determination of airflow resistance, 268 International Organization for Standardization, Geneva, Switzerland.

269 [16] M. Vercammen, Improving the accuracy of sound absorption measurement according to ISO

270 354, in Proc. International Symposium on Room Acoustics, Melbourne, Australia, 2010.

271 [17] Jeong C.-H. Kurtosis of room impulse responses as a diffuseness measure for reverberation

272 chambers. ,’ J. Acoust. Soc. Am. 2016;139:2833-2841.

273 [18] Epain N, Jin CT. Spherical harmonic signal covariance and sound field diffuseness. IEEE/ACM 274 Trans. Audio, Speech, Lang. Process. 2016;10:1796-1807.

275 [19] Chazot J.-D., Robin O, Atalla N, Guyader J.-L. Diffuse acoustic field produced in reverberant 276 rooms : a boundary diffuse field index . Acta Acust. Acust. 2016;102:503-516.

277 [20] Jeong C.-H, Choi S.-H, Lee I. Bayesian inference of the flow resistivity of a sound absorber and 278 the room's influence on Sabine absorption coefficients. J. Acoust. Soc. Am. 2017; 141:1711-1714. 